retrograde transarterial catheterization, or if additional access to the endoleak was required, a direct approach was employed to catheterize the endoleak, including translumbar, transabdominal and perigraft approaches. The latter approach involves undermining the endograft at an iliac limb apposition site. Patients were followed by CT to assess for persistent/recurrent endoleaks and progression of AAA cross-sectional area.

Results: Thirty-six consecutive patients (26 male, mean age 81) were enrolled in the study. Mean time from EVAR to the index embolization procedure was 45.6 months. The transarterial, direct, and perigraft approaches were used in 15, 14, and 13 cases, respectively. Adjunctive coil embolization was performed in 21 cases. No major adverse events were recorded. Twentytwo of $37(59.5 \%)$ cases showed resolution of the endoleak at initial post-procedural CT (mean 2 months). Four patients subsequently developed recurrent type II endoleaks (mean 7 months from index procedure), while 1 patient developed a type I endoleak (11 months). At final follow-up CT (mean 11.3 months), $52 \%$ of patients showed stability $\left( \pm 5 \mathrm{~cm}^{2}\right)$ or decrease $\left(\geq 5 \mathrm{~cm}^{2}\right)$ in the maximal cross-sectional AAA area. Conclusions: EVOH embolization is a safe and effective method for the prevention of aneurysm sac expansion in patients with type II endoleaks post EVAR. We found a trend over time, of abandoning the cumbersome transarterial retrograde lumbar artery catheterization and direct translumbar approaches in favor of the more efficient perigraft and direct transabdominal approaches.

\section{3:36 PM}

Abstract No. 395

\section{Comparison of type II endoleak embolizations: embolization of endoleak nidus only versus} embolization of endoleak nidus and branch vessels H. Yu ${ }^{1}$, M. Al-Roubaie ${ }^{1}$, H. Desai ${ }^{1}$, A. Isaacson ${ }^{1}$, C. Burke ${ }^{1}$; 'University of North Carolina, Chapel Hill, NC

Purpose: To compare outcomes of type II endoleak repair involving embolization of the endoleak nidus only versus embolization of the endoleak nidus and branch vessels in patients with endovascular repair for abdominal aortic aneurysm.

Materials: Thirty-one patients (mean age, 76.7; range, 59-88 years) with 38 type II endoleak repairs between January 2004 and December 2015 were retrospectively analyzed. Procedures for type II endoleak repair were divided into two groups: embolization of the endoleak nidus only (group A) and embolization of both the endoleak nidus and branch vessels (group B). Follow-up CT was evaluated for persistent endoleak, change in the sac diameter, and sac growth rate. Procedure-related complication, procedure time, and patient radiation exposure were recorded. Outcomes were compared using Mann-Whitney U and Pearson's chi-squared tests.

Results: Mean follow-up was $17.3 \pm 13$ months. For groups A and $\mathrm{B}$, persistent endoleak was identified in $66.7 \%$ and $41.2 \%$ $(\mathrm{p}=0.213)$ and the sac decreased or stabilized in $71.4 \%$ and $76.5 \% \quad(p=0.726)$, respectively. There was no significant difference in the rate of persistent endoleak, change in the sac diameter, or sac growth rate. There were no procedure-related complications. Procedure times and radiation exposures were significantly higher in group B $(131 \pm 55.9$ minutes, $20892.5 \pm$ $\left.9078.1 \mu \mathrm{Gym}^{2}\right)$ compared with group $\mathrm{A}(86.2 \pm 41$ minutes, $\left.6455.1 \pm 3950.2 \mu \mathrm{Gym}^{2}\right)(\mathrm{p}=0.03, \mathrm{p}<0.001$, respectively).

Conclusions: There is no significant difference in outcomes between embolization of the endoleak nidus only versus embolization of both the endoleak nidus and branch vessels in patients with type II endoleak, while there is increased procedure time and patient radiation exposure for branch vessel embolization.

\section{3:45 PM}

Abstract No. 396

\section{Evaluating the use of CEUS in the characterization of complex type II endoleaks in patients who underwent failed endoleak repairs} E. Nzekwu ${ }^{1}$, B. Stebner ${ }^{1}$, R. Choo ${ }^{2}$, D. Bakshi ${ }^{3}$, O. Halliwell ${ }^{4} ;{ }^{1}$ University of Calgary, Calgary, Alberta; ${ }^{2}$ University of Calgary, Edmonton, Alberta; ${ }^{3}$ Japanese Red Cross Musashino Hospital, Tokyo, Japan; ${ }^{4}$ N/A, Calgary, Alberta

Purpose: Endovascular Aneurysm Repair (EVAR) is a safe and efficacious treatment for abdominal aortic aneurysms (AAA). However, patients must undergo lifelong surveillance following EVAR to evaluate for the presence of complications, including endoleaks. Computed tomography angiography (CTA) is the contemporary gold-standard imaging modality for surveillance of EVARs but involves a cumulative radiation exposure, and a risk of contrast-induced nephropathy. Small endoleaks are sometimes undetectable with CTA. Early reports on contrast enhanced ultrasound (CEUS) have demonstrated favorable results with sensitivities and specificities reportedly non-inferior to CTA. This retrospective review aims to evaluate the use of CEUS in patients undergoing re-intervention post-endoleak repair.

Materials: Between April 2010 and December 2015 a retrospective review was conducted on 98 patients in 2 major tertiary hospitals undergoing CEUS imaging for the evaluation of Type II endoleaks post-EVAR. Inclusion criteria selected for patients who underwent a failed endoleak repair post-EVAR. CEUS was subsequently utilized to further characterize the endoleak $(n=14)$ compared with recent CTA examinations. The efficacy of CEUS was then evaluated.

Results: 14 patients met the inclusion criteria with CEUS further characterizing the endoleak in $12(86 \%)$ of cases when compared with CTA, ultimately contributing to future management. In 7 $(50 \%)$ cases, CEUS altered management, including specifying involved feeding vessels to type II endoleaks or modifying the eventual approach used for future embolization. In $1(7 \%)$ of the cases, CEUS was discordant with CTA findings resulting in subsequent angiography. Technical success was eventually achieved in $13(93 \%)$ of patients despite difficult anatomy/ persistent leaks, with 1 case failing secondary to patient body habitus. CEUS discovered endoleaks missed by a follow-up CTA in $3(21 \%)$ different patients at various stages of surveillance.

Conclusions: CEUS is an effective imaging modality to supplement CTA findings particularly in complex endoleak cases further characterizing leaks and delineating aneurysmal morphology, ultimately altering treatment approaches 